\title{
Enhancement of nucleon-nucleon cross section in sidewards flowing nuclear matter through zero sound mode excitation
}

\author{
J. Diaz-Alonso*, L. Mornas ${ }^{\dagger}$ \\ *DARC, UPR 176 CNRS, Observatoire de Paris - Meudon, F-92195 Meudon, France \\ and Universidad de Oviedo, Departamento de Física, E-33007 Oviedo, Spain \\ ${ }^{\dagger}$ Departamento de Física Teórica, Universidad de Valencia, 46100 Burjasot (Valencia), Spain
}

February 12, 2018

PACS: 25.40.Cm, 21.30.-x, 21.65+f, 24.10.Jv, 21.60.Jz

keywords: in-medium NN cross section, screened one-boson exchange,relativistic RPA, zero-sound

\begin{abstract}
We investigate the modification induced on the nucleon-nucleon cross section by screening of the interaction inside nuclear matter. The interaction is described by the relativistic one boson exchange of $\sigma, \omega$ and $\pi$ mesons. Medium effects enter as loop corrections to the meson propagators and are characterized not only by density, but also by temperature and velocity with respect to the center of mass of the collision. The cross section displays peaks for some specific values of the velocity, corresponding to the excitation of zero-sound modes of the longitudinal $\omega$ field. The enhancement factor amounts to about 1.5 under reasonable conditions. It increases with density and is reduced at finite temperature. These findings may have verifiable consequences on the angular dependence of the measurable secondary particle distributions.
\end{abstract}

\section{Introduction}

Dissipative effects play an important role in explaining the shapes of the angular distributions and energy spectra of the secondary particles produced in a relativistic heavy ion collision. For this reason, the cross section is an important ingredient in all kinds of numerical simulations of these collisions, be it in the molecular dynamics approach [1, 2], kinetic theory approaches [3, 4], or the hydrodynamical models where it enters through the transport coefficients [5]. It has become obvious in the last years that using the free value of the cross section does not well reproduce the available experimental data; rather, the medium may affect the nucleonnucleon cross section by modifying the strength of the interaction and the available phase space.

Several attempts to calculate medium corrections to the cross section already exist. The first point to precise is in what type of background matter does the collision take place. Some authors like Faessler et al. [6] or Fuchs et al. [7] consider that the background consists of a momentum distribution in two well separated Fermi spheres. This corresponds to the initial stage of a heavy ion collision and is far out of equilibrium. Other authors like Machleidt et al. [8] or Alm et al. [9] consider that the background is nuclear matter, supposedly of infinite extension, and not far from equilibrium. This rather corresponds to the intermediate stage of the collision after a fireball was formed. This latter case is what we are interested in here. 
Medium effects enter at several levels of the calculation of the cross section. Older evaluations only took into account the shifting of the nuclear mass to an effective smaller value inside nuclear matter. Then, in the last few years were performed a number of calculations with the Brueckner formalism [8, 9, 10]. In this approach, an infinite series of ladder diagrams are resummed. The nucleon propagators, i.e. the uprights of the ladder, are dressed. The medium effects enter as Pauli blocking factors on the intermediate states in the inner nucleon lines. The meson lines are the free ones, corresponding to an unscreened interaction.

On the other hand, we would like to discuss in this letter the influence of the screening of the interaction by the surrounding nuclear matter. In a model where the $\mathrm{N}-\mathrm{N}$ interaction is described by relativistic meson exchanges, we take into account the dressing of the meson lines in the first Born approximation. A detailed study of the density and temperature dependence of the total and differential elastic $p$ - $p$ cross section was performed in our first paper [11]. It was assumed there that the background nuclear matter was at rest with respect to the center of mass of the collision. The concern of our present work is to release this assumption. We refer the reader to [11] for details on the formalism and only recall here the main formulaes.

\section{The model}

We are working with the standard quantum hadrodynamics lagrangian with $\sigma, \omega$ and $\pi$ meson exchange. This is the minimal model which permits to reproduce the available experimental data on $p-p$ scattering to a satisfactory level of accuracy. The interaction lagrangian reads

$$
\mathcal{L}=g_{\sigma} \bar{\psi} \sigma \psi+g_{\omega} \bar{\psi} \gamma^{\mu} \omega_{\mu} \psi+g_{\pi} \bar{\psi} \gamma^{5} \vec{\tau} \vec{\pi} \psi
$$

The differential elastic cross section was calculated in the Born approximation. In the center of mass of the collision,

$$
d \sigma / d \Omega=1 /\left(64 \pi^{2} s\right)|\mathcal{M}|^{2}
$$

where $s$ is the Mandelstam variable $s=4 E_{c . m}^{2}$. and the transition matrix is given by

$$
\mathcal{M}=\sum_{\mathrm{m}, \mathrm{n}=\sigma, \omega, \pi}\left(\bar{U}_{3} \Gamma_{m} U_{1}\right) G_{31}^{\mathrm{mn}}\left(\bar{U}_{4} \Gamma_{n} U_{2}\right)-\text { exchange }
$$

In this expression the $U$ 's are the spinors defining the incoming $\left(U_{1}, U_{2}\right)$ and outgoing $\left(U_{3}, U_{4}\right)$ nucleon states. The indices $i=1,2,3,4$ carried by the spinors stand for the momenta $p_{i}^{\mu}$ and the spin-isospin quantum numbers $s_{i}, t_{i} . G_{31}^{\mathrm{mn}}$ is the propagator matrix which depends on the momentum transfer $k_{31}^{\mu}=p_{1}^{\mu}-p_{3}^{\mu}$. In free space, it reduces to the standard form

$$
G_{31}^{\mathrm{mn}}=\operatorname{Diag}\left(\frac{-i\left[g^{\mu \nu}-\frac{k^{\mu} k^{\nu}}{k^{2}}\right]}{k^{2}-m_{\omega}^{2}}, \frac{i}{k^{2}-m_{\sigma}^{2}}, \frac{i}{k^{2}-m_{\pi}^{2}}\right)
$$

$\Gamma$ is the coupling matrix $\Gamma=\left(-i g_{\omega} \gamma^{\mu}, i g_{\sigma}, g_{\pi} \gamma_{5} \vec{\tau}\right)$. In the actual calculation of the transition matrix, all coupling constants are multiplied by form factors $\left(\Lambda_{\mathrm{m}}^{2}-m_{\mathrm{m}}^{2}\right) /\left(\Lambda_{\mathrm{m}}^{2}-k^{2}\right)$. The exchange term is obtained by interverting indices 3 and 4 . In the following we consider only the spin-averaged cross section $\overline{|\mathcal{M}|^{2}}=(1 / 4) \sum_{s_{i}}|\mathcal{M}|^{2} . \sigma_{p p}$ and $\sigma_{n n}$ are equal in our approximation where the Coulomb interaction was substracted and the neutron and proton masses are equal. We do not show $\sigma_{n p}$ since our simple model does not include $\rho$ meson exchange.

In paper I we performed a fit of the free elastic $p$ - $p$ cross section using the available experimental data [12]. We kept constant the masses of the mesons and the pion-nucleon 
coupling $\left(m_{\sigma}=550 \mathrm{MeV}, m_{\omega}=783 \mathrm{MeV}, m_{\pi}=550 \mathrm{MeV}, g_{\pi}^{2} / 4 \pi=14.4\right)$ and fitted the coupling constants $g_{\sigma}, g_{\omega}$ and the cutoffs $\Lambda_{\sigma}, \Lambda_{\omega}, \Lambda_{\pi}$. Several sets of parameters were found to reproduce the data to a satisfactory level of accuracy. It was checked that the in-medium behavior of the cross section was the same for all choices of the parameter set. The calculations are performed here with parameter set A of paper I: $g_{\sigma}=3.80, g_{\omega}=9.31, \Lambda_{\sigma}=1298.8$, $\Lambda_{\omega}=1240.5, \Lambda_{\pi}=362.1$.

Inside the medium, the momenta and masses of the incoming and outgoing nucleons must be replaced by their effective counterparts. In the Hartree approximation, $p_{i}^{\mu} \rightarrow\left(p_{i}^{\mu}\right)^{*}=p_{i}^{\mu}-$ $g_{\omega}\left\langle\omega^{\mu}\right\rangle, m \rightarrow m_{*}=m-g_{\sigma}\langle\sigma\rangle$. The propagator matrix must also be modified in order to take into account that the N-N interaction is mediated by dressed mesons. The dispersion relations and propagators of the mesons at finite temperature were obtained from a linear kinetic analysis of perturbations around the Hartree ground state [13]. The results of this method coincide at $T=0$ with those of the one-loop approximation [14]. The calculation takes into account renormalized vacuum polarization contributions which are crucial in obtaining a physically reasonable behavior of the propagation modes 13. The propagator matrix so obtained has the form [11, 14, 15].

$$
G^{m n}=\left(\begin{array}{ccc}
G_{\omega}^{\mu \nu} & G_{\sigma \omega}^{\mu} & 0 \\
G_{\omega \sigma}^{\mu} & G_{\sigma} & 0 \\
0 & 0 & G_{\pi}
\end{array}\right)
$$

$$
\text { with } \begin{aligned}
G_{\omega}^{\mu \nu} & =-i\left[\mathcal{T}^{\mu \nu} \frac{1}{k^{2}-m_{\omega}^{2}+g_{\omega}^{2} \Pi_{\omega T}}+\Lambda^{\mu \nu} \frac{k^{2}-m_{\sigma}^{2}+g_{\sigma}^{2} \Pi_{\sigma}}{D_{\sigma-\omega L}(k)}-\frac{k^{\mu} k^{\nu}}{m_{\omega}^{2} k^{2}}\right] \\
G_{\sigma} & =i \frac{k^{2}-m_{\omega}^{2}+g_{\omega}^{2} \Pi_{\omega L}}{D_{\sigma-\omega L}(k)} ; \quad G_{\sigma \omega}^{\mu}=-i \eta^{\mu} \frac{g_{\sigma} g_{\omega} \Pi_{\times}}{D_{\sigma-\omega L}(k)} \\
G_{\pi} & =i \frac{1}{k^{2}-m_{\pi}^{2}+g_{\pi}^{2} \Pi_{\pi}}
\end{aligned}
$$

In these expressions we defined the quadrivector $\eta^{\mu}$ and the projectors onto the longitudinal $\left(\Lambda^{\mu \nu}\right)$ and transverse $\left(\mathcal{T}^{\mu \nu}\right)$ modes as functions of the 4 -velocity $u^{\mu}$ of the fluid as follows:

$$
\begin{aligned}
& \eta^{\mu}=u^{\mu}-\frac{k \cdot u}{k^{2}} k^{\mu} \quad ; \quad \tilde{g}^{\mu \nu}=g^{\mu \nu}-\frac{k^{\mu} k^{\nu}}{k^{2}} \\
& \mathcal{T}^{\mu \nu}=\tilde{g}^{\mu \nu}-\frac{\eta^{\mu} \eta^{\nu}}{\eta^{2}} \quad ; \quad \Lambda^{\mu \nu}=\frac{\eta^{\mu} \eta^{\nu}}{\eta^{2}}
\end{aligned}
$$

The polarizations $\Pi(k)$ of the meson fields are functions of the thermodynamical state (density and temperature) of the matter and on the transferred 4-momentum $k^{\mu}=(\omega, \vec{q})$. There is a mixing between the $\sigma$ and longitudinal- $\omega$ propagation modes. The $\pi$ propagation is decoupled from the dynamics of the other mesons in symmetric nuclear matter. We use the notation

$$
\Pi_{\times}=\eta_{\mu} \Pi_{\sigma \omega}^{\mu} \quad ; \quad \Pi_{\omega L}=-\Lambda_{\mu \nu} \Pi_{\omega}^{\mu \nu} \quad ; \quad \Pi_{\omega T}=-\mathcal{T}_{\mu \nu} \Pi_{\omega}^{\mu \nu} / 2
$$

for the mixing, longitudinal and transverse parts. The explicit expression for the polarizations and a study of the dispersion relations can found in [11, 13]. Besides the $\sigma$ and longitudinal and transverse $\omega$ branches, there is a zero sound branch in the mixed $\sigma-\omega$ mode.

The denominator of the $\sigma$, longitudinal part of the $\omega$ and mixing propagators is given by the mixed $\sigma-\omega$ dispersion relation

$$
D_{\sigma-\omega L}(k)=\left(k^{2}-m_{\sigma}^{2}+g_{\sigma}^{2} \Pi_{\sigma}\right)\left(k^{2}-m_{\omega}^{2}+g_{\omega}^{2} \Pi_{\omega L}\right)+g_{\sigma} g_{\omega} \Pi_{\times}^{2}
$$


The polarizations are complex. In the $T=0$ limit, the imaginary part of the polarizations vanish outside the region defined by

$$
\sqrt{\left(p_{F}-q\right)^{2}+m_{*}^{2}}-\sqrt{p_{F}^{2}+m_{*}^{2}}<\omega<\sqrt{\left(p_{F}+q\right)^{2}+m_{*}^{2}}-\sqrt{p_{F}^{2}+m_{*}^{2}}
$$

(where $p_{F}$ is the Fermi momentum and $m_{*}$ the effective mass of the nucleon inside the medium). Inside this region, the propagation modes are damped by the decay into particlehole pairs. At finite temperature the imaginary parts are finite for any space-like mode.

The dependence on the velocity of the medium comes through the quadrivector $\eta^{\mu}$. In the referential (R1) where the fluid is at rest, $u^{\mu}=(1, \overrightarrow{0})$ and the momentum transfer is $k^{\mu}=(\omega, \vec{q})$

In the center of mass of the collision (referential (R2)), the background fluid moves with a velocity $u^{\mu}=(\gamma, \gamma \vec{v})$ parametrized by

$$
\vec{v}=(v \sin \alpha \sin \varphi, v \sin \alpha \cos \varphi, v \cos \alpha)
$$

The momentum transfers for the direct $\left(k_{31}\right)$ and exchange $\left(k_{41}\right)$ diagrams are

$$
k_{31}^{\mu}=(0,0, p \sin \theta, p(1-\cos \theta)) \quad ; \quad k_{41}^{\mu}=(0,0,-p \sin \theta, p(1+\cos \theta))
$$

with $p=\sqrt{E_{c . m}^{2}+m_{*}^{2}}$ and $\theta$ is the $c . m$. scattering angle.

One goes from (R1) to (R2) by performing a boost of four-velocity $(\gamma, \gamma \vec{v})$ and a rotation. In the referential $(\mathrm{R} 1)$,

$$
\begin{aligned}
k_{31}^{\mu}(R 1) & =\left(\omega_{\ominus}, \vec{q}_{\ominus}\right) \quad ; \quad k_{41}^{\mu}(R 1)=\left(\omega_{\oplus}, \vec{q}_{\oplus}\right) \\
\omega_{(\underset{\oplus}{\ominus})} & =\gamma v p[ \pm \sin \alpha \cos \varphi \sin \theta-\cos \alpha(1 \mp \cos \theta)] \\
q_{(\underset{\oplus}{\ominus})} & =\left[\omega_{(\underset{\oplus}{\ominus})}^{2}+2 p^{2}(1 \mp \cos \theta)\right]^{1 / 2}
\end{aligned}
$$

\section{In medium cross section for finite relative velocity}

When the rest frame of the background nuclear matter is moving with respect to the center of mass of the colliding particles, the relative velocity will enter the expression of the cross section through the meson propagators. There is an explicit dependence coming from the projectors $\Lambda^{\mu \nu}, \mathcal{T}^{\mu \nu}$ (see Eq. (7)). The projectors act by attributing velocity-dependent weights to the various components of the currents $\dot{\mathcal{J}}$ like e.g. $\dot{\mathcal{J}}_{\omega}^{\mu \nu}=\left(\bar{U}_{3} \gamma^{\mu} U_{1}\right)\left(\bar{U}_{4} \gamma^{\nu} U_{2}\right)$. There is also an indirect dependence in the velocity in the expression of the polarizations $\Pi\left(\omega_{(\underset{\oplus}{\ominus})}, q_{(\underset{\oplus}{\ominus})}\right)$ coming from the boost transformation Eq. (13). In contrast to the zero velocity case where the momentum transfer $k^{\mu}$ entering the expression of the polarizations was confined to the

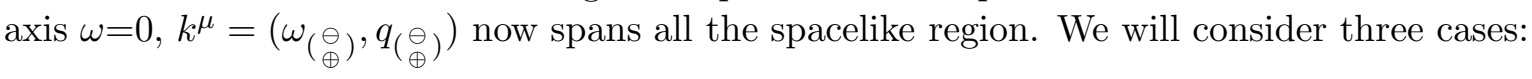

(i) $v=\left(0,0, v_{z}\right)$

For moderate values of the velocity along the axis defined by the incident particles $\left(v=v_{z}\right)$, the cross section is slightly enhanced at low densities and reduced at high densities. When some temperature is introduced, the low density enhancement disappears and the high density reduction persists. At highly relativistic $v_{z}$ velocities $\left(\gamma_{z} \gtrsim 5\right)$, the cross section displays a series of peaks, of which three are clearly visible. The height of the peaks increases with density. The effect of finite temperature is to gradually wash out these peaks. The peaks are superposed on an additional increase of the cross section, which stabilizes to a large constant value for large $\gamma_{z}$. This additional enhancement persists when introducing finite temperature. 
(ii) $v=\left(0, v_{y}, 0\right)$

For low values of the velocity inside the reaction plane and orthogonal to the beam axis $\left(v=v_{y}\right)$, the cross section is first approximately constant for densities up to $n=n_{0}$ and somewhat reduced at high density when increasing $v_{y}$. Further increasing $v_{y}$, one meets a peak structure (see Fig. 1) superposed on a smooth increase similar to the one already observed in the case $v=v_{z}$, but occurring for more physical values of the velocity. There is first a sharp peak for a characterictic value $\hat{v}_{y}$ immediately followed by one or two smoother peaks. Further increasing the velocity, the cross section slowly decreases and stabilizes to a constant value $\sigma_{\text {med }} / \sigma_{\text {free }}\left(v_{y} \rightarrow c\right)$ somewhat larger than $\sigma_{m e d} / \sigma_{\text {free }}(v=0)$ and approximately equal to 1 . We were able to check that the peak structure originates from the velocity dependence of the polarizations while the residual temperature-independent enhancement at $v_{y} \rightarrow c$ is due to the velocity dependence of the projectors $\Lambda^{\mu \nu}, \mathcal{T}^{\mu \nu}$. The same holds for the case $v / / z$.

(iii) $v=\left(v_{x}, 0,0\right)$

No appreciable modification is observed for velocities orthogonal to the reaction plane $(v=$ $\left.v_{x}\right)$ up to $v_{x} \simeq 0.8 c$. On the other hand, the total cross section starts to increase above this value and continues to grow indefinitely as $\gamma_{x} \rightarrow \infty$.

The angular dependence of the differential cross section deviates appreciably from its behavior when the fluid is at rest. For each value of the velocity, there is a favoured angle, but this effect is most appreciable for small angles, so that finite velocities also contribute to make the cross section more forward/backward peaked with respect to its vacuum value.

We concentrate on the behaviour with $v_{y}$ which is the case most liable to lead to observable consequences since the peaks appear for reasonable values of the relative velocity. The characteristic velocity $\hat{v}_{y}$ at which the sharp peak takes place is density dependent, e.g. one has $\hat{v}_{y} \simeq 0.41 c$ for $n=n_{0}$ and $\hat{v}_{y} \simeq 0.57 c$ for $n=2 n_{0}$ (see Fig. 1a). The height of the peak increases with increasing density and is reduced by increasing the temperature (see Fig. 1b). At $T=10 \mathrm{MeV}$, a broad bump is still clearly visible around $\hat{v}_{y}$, at high temperature $T \gtrsim 50$ - $100 \mathrm{MeV}$ it has completely disappeared.

The peak structure is to be related to the excitation of the zero sound modes. These modes arise from the mixed $\sigma$ - longitudinal $\omega$ part of the dispersion relation; they were studied in previous work [13, 15]. A contour plot of the mixed dispersion relation $D_{\sigma-\omega L}(\omega, q)$ in the $q-\omega$ plane of transferred momentum is shown in Fig. 2 for $T=0, n=n_{0}$. The level $D_{\sigma-\omega L}(\omega, q)=0$ gives the location of the zero-sound branch. For not too high values of the energy-momentum transfer, the region of the zero sound branches can be crossed for some values of the velocity in the medium: If $(\omega, q)$ coincides with a zero-sound solution $k_{z s}^{\mu}=\left(\omega_{z s}, q_{z s}\right)$ of the dispersion relation $D_{\sigma-\omega L}\left(k_{z s}^{\mu}\right)=0$, there will be a critical value of the relative velocity $\hat{v}$ solution of

$$
\omega_{z s}=\omega_{(\underset{\oplus}{\ominus})}=\gamma \hat{v} p[ \pm \sin \alpha \cos \varphi \sin \theta-\cos \alpha(1 \mp \cos \theta)]
$$

(cf. Eqs. $(9,13))$ for which the real part of the dispersion relation vanishes. Since the dispersion relation enters in the denominator of the propagators ( $c f$. Eq. (7)), a pole appears in the expression of the cross section ( $c f$. Eqs. (2. 3) ) if the imaginary part of $D_{\sigma-\omega L}$ vanishes as well. For velocities near $\hat{v}, D_{\sigma-\omega L}$ is still reduced and as a consequence, the cross section enhanced.

For example, at $n=n_{0}$ and $T=0$, we have drawn on Fig. 2 the path swept by the components of momentum transfer for fixed laboratory energy $\left(T_{l a b}=300 \mathrm{MeV}\right)$ and relative velocity as the integration needed to calculate the total elastic in-medium cross section is carried out. It is seen that it falls on the upper part of the zero sound branch for $\hat{v}_{y}=0.41$ corresponding to the location of the peak in Fig. 1. 
The lower branch of the zero sound falls in the particle-hole damping zone. On the other hand, part of the upper branch falls outside the particle-hole damping zone where the imaginary part of $D_{\sigma-\omega L}$ vanishes corresponding to undamped modes at our level of approximation. This is at the origin of the narrowest peaks shown in Fig. 1, while the smoother peak is to be related to the lower branch for which particle/hole damping is active. The zero-sound branches become more important as the density increases above a threshold value, their importance is reduced as temperature increases. It is indeed to be seen in Fig. 1a that the peak structure begins to develop above $n=0.5 n_{0}$ and in Fig. $1 \mathrm{~b}$ that it is washed out at high temperature.

\section{Discussion and conclusion}

In a relativistic OBE model of the $\mathrm{N}-\mathrm{N}$ interaction with $\sigma, \omega$ and $\pi$ meson exchange, we investigated the observable consequences of a modification of the elastic proton-proton cross section inside symmetric nuclear matter by screening of the interaction, i.e. by approximating the meson propagators at the RPA level. In particular we concentrated on the influence of a non vanishing relative velocity between the center of mass of the collision and the rest frame of the fluid. The velocity dependence was found to be non monotonous. For moderate values of the velocity, the cross section is enhanced at low densities and reduced at high densities if the velocity is inside the reaction plane $(y, z)$ and no appreciable modification is observed for velocities orthogonal to the reaction plane $(v / / x)$.

The main result of our calculations is the enhancement introduced by the excitation of the zero sound modes of the medium on both the differential and the total elastic cross sections. A peak appears for a charateristic value of the relative velocity. Its location is density dependent; the peak is stronger at higher density and gradually blurs out when increasing temperature. It occurs for $v / / y$ at physically relevant values $0.3<\hat{v}_{y}<0.8$. but not for $v / / x$ or for unrealistic values of $v / / z$.

Such an asymmetric configuration has not yet been considered up to our knowledge. A selective enhancement of the cross section for a well defined value of the density and velocity will probably have a characteristic signature in terms of the transverse flow and angular distributions. In a naive hydrodynamical picture, we found that the collisions are more efficient when they take place in a background matter flowing in the direction corresponding to the "sidewards flow", moreover the scattered products are predominantly directed backward and forward. However, it is delicate at this stage of our investigations to put forward a definite prediction on the consequences on experimental observables, since they are the result of the interplay of many factors. For example, it is known that viscous effects [5] or the softening of the equation of state (EOS) through a possible phase transition to the quark gluon plasma 116 may reduce the directed sidewards flow. We think that our findings may bring new arguments in the context of the anisotropic flow observed recently both in $\mathrm{Au}+\mathrm{Au}$ collisions at $100-800 \mathrm{MeV} / \mathrm{c}$ at the FOPI facility at GSI Darmstadt and by the E877 collaboration at AGS in $\mathrm{Au}+\mathrm{Au}$ collisions at $10.8 \mathrm{GeV} / \mathrm{c}$. The anisotropy is not yet fully understood [17] and has been analysed mainly in terms of the EOS. It could be worthwhile to investigate the effect of an anisotropically enhanced cross section in this kind of numerical simulations.

The enhancement we have found only depends on the reduction of the real part of the dispersion relation associated with the vicinity to a zero sound mode in the matter. The existence of a zero-sound branch in nuclear matter is well documented (see e.g. [13, 19]), so that our result is a quite general one. Its size depends on the parameters, in particular on the cutoffs $\Lambda_{\sigma}$ and $\Lambda_{\omega}$. Our parameters come from a fit of the free cross section; the cutoffs $\Lambda \simeq 1200 \mathrm{MeV}$ and coupling constants are rather lower those of the Bonn potential, so that 
we feel we are on the safe side. Let us say a few words on higher order corrections. Vertex functions such as calculated by Allendes and Serot [18] are amenable at low and intermediate momentum transfer to a monopole form factor with $\Lambda \simeq 1100 \mathrm{MeV}$. If the coupling constants are decreasing functions of the density as argued from Dirac-Brueckner-Hartree-Fock calculations, the density dependence of our enhancement can be modified. (The determination of a more accurate density dependence would require a fully consistent calculation of ladders as well as loops which is not yet available.) Even if these branches would disappear completely (by applying very low cutoffs), there would still be a strong reduction of the dispersion relation $D_{\sigma-\omega L}(\omega, q)$ in this zone due to $\sigma-\omega$ mixing and consequently an enhancement in the cross section. We believe that this broad enhancement at finite relative velocity matters more than the presence of a high but narrow peak. Its location will still be determined by the upper limit of the particle hole damping zone.

Finally, we would like to warn the reader against a possible confusion with an enhancement found by Alm el al. [9] in an other context. These authors perform a nonrelativistic Brueckner calculation. Their enhancement is caused by a pole in the propagator of the intermediate two-nucleon state; the result is interpreted as a precursor of superfluidity. The enhancement occurs for low values of the density $n / n_{0} \simeq 0.5$; the peak is reduced and finally disappears when the total momentum $K$ of the pair deviates from zero. This is clearly different from our case where the enhancement is due to a pole in the mixed meson propagator and occurs for high values of the density equal or larger than $n / n_{0}=0.5$ and for nonvanishing values of the velocity $v / c=0.3-0.7$.

\section{References}

[1] D.T. Khoa et al., Nucl. Phys. A548 (1992) 102.

[2] E.I. Tanaka, et al., Phys. Rev. C52 (1995) 316.

[3] G.D. Westfall et al., Phys. Rev. Lett. 71 (1993) 1986; V. de la Mota et al., Phys. Rev. C46 (1992) 677; B.A. Li, Phys. Rev. C48 (1993) 2415.

[4] D. Klakow, G. Welke and W. Bauer, Phys. Rev. C48 (1993) 1982.

[5] see e.g. R.B. Clare and D. Strottman, Phys. Rep. 141 (1986) 177; H.H.K. Tang and C.Y. Wong, Phys. Rev. C21 (1980) 1846, ibid C26 (1982) 284; W. Schmidt et al., Phys. Rev. C47 (1993) 2782.

[6] A. Bohnet et al., Nucl. Phys. A494 (1989) 349; A. Faessler, Nucl. Phys. A495 (1989) $103 \mathrm{c}$.

[7] C. Fuchs, L. Sehn and H.H. Wolter, Nucl. Phys. A601 (1996) 505.

[8] G.Q. Li and R. Machleidt, Phys. Rev. C48 (1993) 1702.

[9] T. Alm, et al., Nucl. Phys. A587 (1995) 815 and Phys. Rev. C50 (1994) 31.

[10] B. ter Haar and R. Malfliet, Phys. Rev. C36 (1987) 1611.

[11] J. Diaz-Alonso, L. Mornas, Nucl. Phys. A629 (1998) 679.

[12] Nijmegen NN-Online Database, V.G.J Stoks et al. Phys. Rev. C47, (1993), 761, ibid. C48 (1993) 792 and SAID program by R.A. Arndt et al. Phys. Rev. C50, 2731 (1994). 
[13] J. Diaz Alonso and A. Pérez Canyellas, Nucl. Phys. A526 (1991) 623.

[14] E. Gallego, J. Diaz Alonso and A. Pérez, Nucl. Phys. A578 (1994) 542; J. Diaz Alonso, E. Gallego and A. Perez, Phys. Rev. Lett. 73 (1994) 2536.

[15] J. Diaz Alonso, B. Friman, P.A. Henning, "Normal Modes in Nuclear Matter at Finite Temperature". Unpublished preprint G.S.I. Darmstadt (1992). Some results of this last reference have been published in P.A. Henning, Phys. Rep. 253 (1995), 235.

[16] D.H. Rischke, Nucl. Phys. A610 (1996) 88c.

[17] P. Crochet et al., FOPI collaboration, Nucl. Phys. A627 (1997) 522; J. Barrette et al., E877 collaboration, Phys.Rev. C55 (1997) 1420.

[18] M.P. Allendes and B.D. Serot, Phys. Rev. C45 (1992) 297.

[19] A.L. Fetter and J.D. Walecka, "Quantum theory of many particle systems", McGrawHill, New York (1971) p. 183ff.; J.C. Caillon, J. Labarsouque, Phys. Lett. B352 (1995) 193.

\section{Figure captions}

Fig. 1: Ratio of the in-medium cross section with respect to its value in the vacuum $\sigma_{\text {med }} / \sigma_{\text {free }}$ as a function of velocity $\left(0, v_{y}, 0\right)$. - (a) for density $n / n_{0}=0.1,0.5,1,2,5-(b)$ temperature dependence at $n=n_{0}$.

Fig. 2: Levels of constant value of the mixed $\sigma$-longitudinal $\omega$ dispersion relation $D_{\sigma-\omega L}(\omega, q)$ in the $q-\omega$ plane of transferred momentum. The long-dashed line $\hat{v}_{y}=0.41$ corresponds to the resonant condition $\hat{\gamma}_{y} \hat{v}_{y} p \sin \theta=\omega_{z s}$. 
Fig. 1a

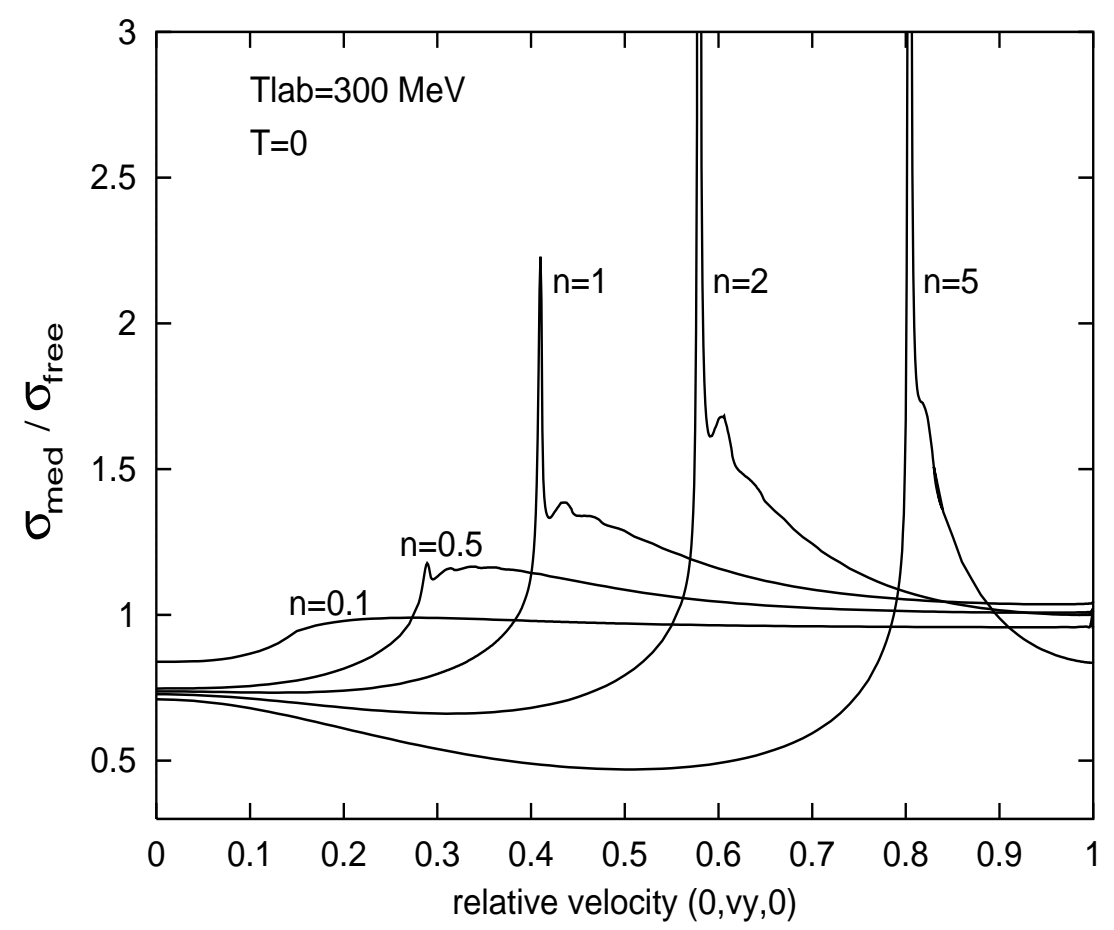

Fig. 1b

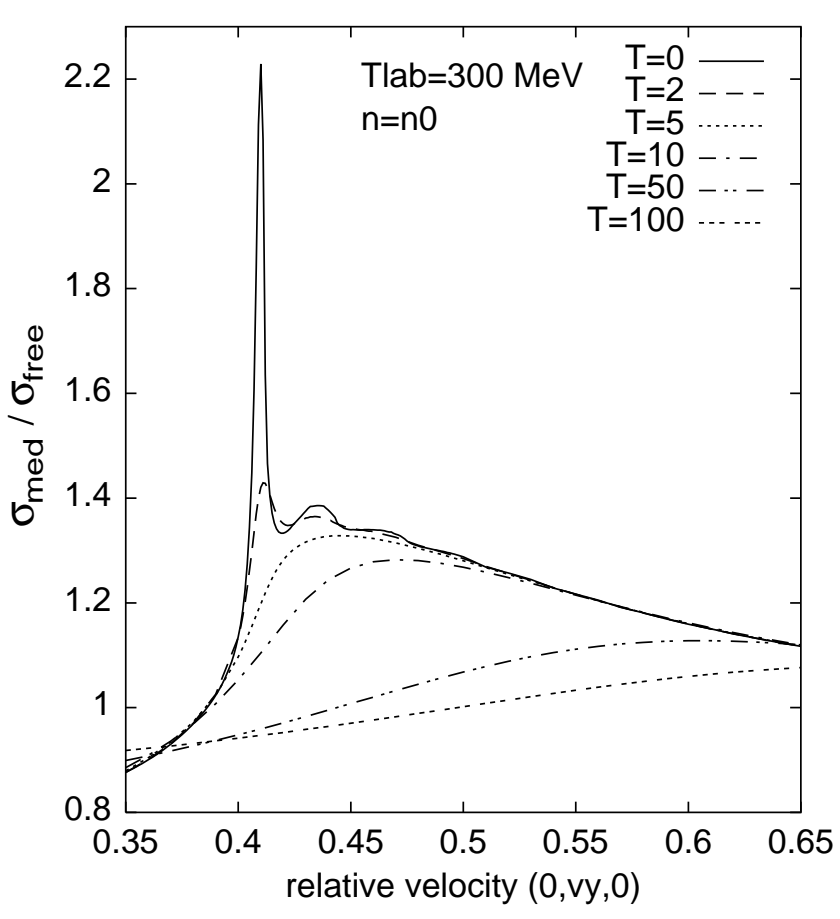


Fig. 2

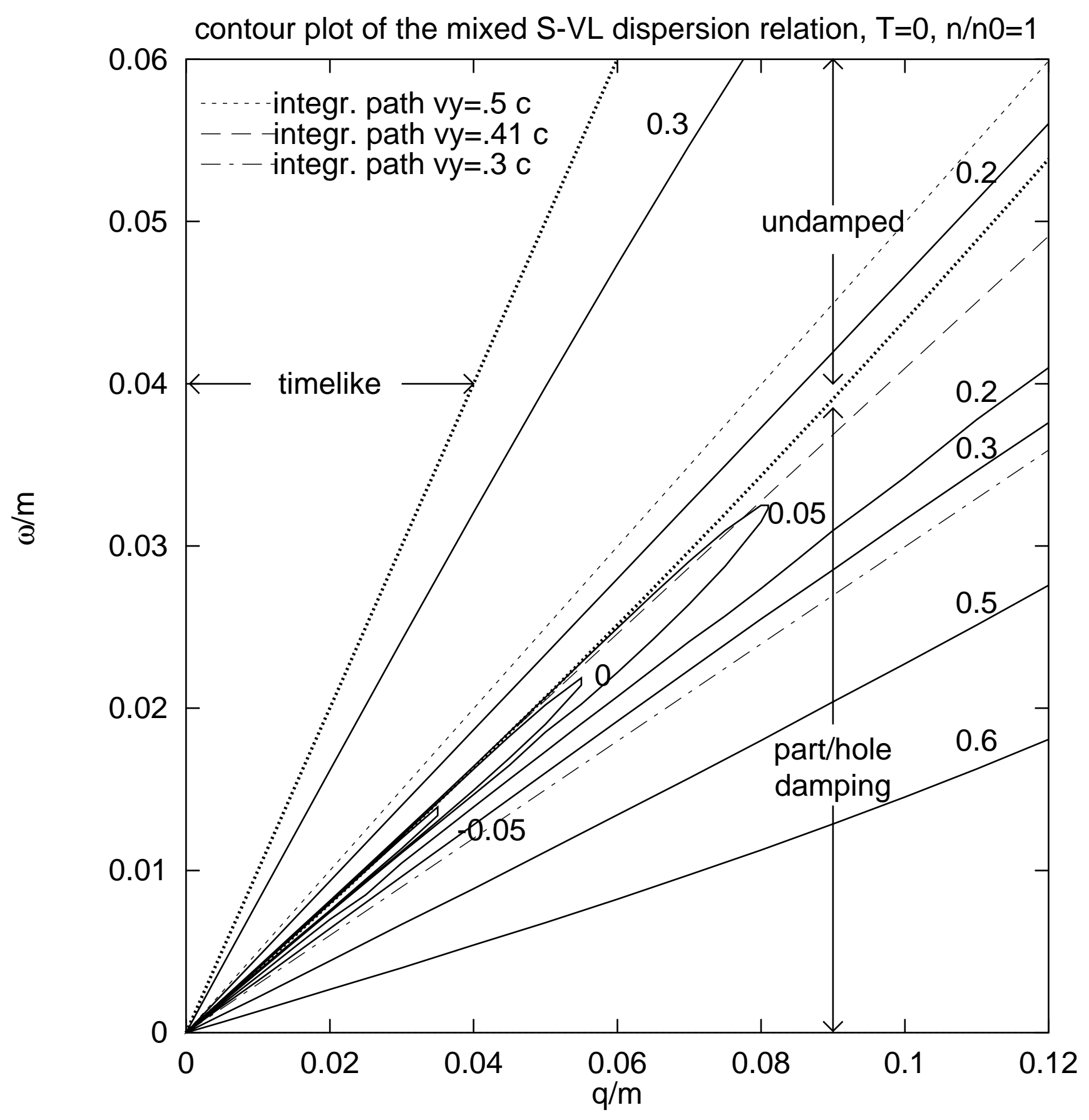

